\title{
Accelerating Unpopular Channel through Federation
}

\author{
Jing $\mathrm{He}^{1,2}$ \\ ${ }^{1}$ School of Computer Science and Engineering \\ University of Electronic Science and Technology of \\ China \\ Chengdu 611731, China \\ E-mail: ynuhj@163.com
}

\author{
Wei Zhou* ${ }^{2}$, Shipu Wang ${ }^{2}$ \\ ${ }^{2}$ National Pilot School of Software \\ Yunnan University \\ Kunming 650091, China \\ E-mail: wz.weizhou@gmail.com, spwang@ynu.edu.cn
}

\begin{abstract}
Nowadays, peer-to-peer technology has provided users with cheap and powerful communicating facilities. However, P2P streaming system faces two challenges. Firstly, how to improve the poor Quality-of-Experience (QoE) in unpopular channel? Secondly, how to select peers to contribute their resources for assisting the unpopular channel in order to balance channel resources? This paper presents a crosschannel federation model to address these problems. The key idea is to identify a set of stable nodes in popular channel to assist the unpopular channel. These stable nodes, together with nodes in unpopular channel, are organized as mesh-based network, which facilitates the unpopular channel to enlarge the channel group and promote peer stability. This cross-channel federation model is evaluated through simulations. The results demonstrate its efficiency.
\end{abstract} model

Keywords-P2P; unpopular channel; cross-channel federation

\section{INTRODUCTION}

The development of new media technologies is providing users with cheap and powerful communicating facilities. PPLive and UUSee released recently have offered more than hundreds of channels. However, the distributions of peers between popular and unpopular channels are quite different. Such unbalanced distribution has inevitably influence the channel performance. In providing multi-channel services, P2P streaming system faces several challenges: (1) How to improve the poor Quality-of-Experience (QoE) in unpopular channel? (2) How to select peers to contribute their resources for assisting the unpopular channel in order to balance channel resources? (3) How to schedule chunk for these assisting peers efficiently and don't influence their original channels' performance?

A possible enhancement to improve the $\mathrm{QoE}$ in unpopular channel is to deploy more video channel servers. However the infrastructure cost grows linearly with user population and video quality. Can we utilize the popular channel to assist the unpopular channel? Actually this is probably the most natural and efficient method in terms of multi-channel resource balance.

In this paper, we propose a cross-channel federation model to address these problems. The key idea is to identify a set of stable nodes in popular channel to assist the unpopular channel. These stable nodes, together with nodes in unpopular channel, are organized as mesh-based network, which facilitates the unpopular channel to enlarge the channel group and promote peer stability. Specifically a series of critical model design challenges are discussed in the paper. To assess the performance of multi-channel federation model, we built a detailed packet-level P2P simulator based on NS2 and conducted extensive simulations. The simulation results demonstrate the efficiency and robustness of this federation solution.

The remainder of this paper is organized as follows. In Section II, we introduce the background and related work. Section III presents our cross-channel federation model. Then, we evaluate the performance of cross-channel federation model in Section IV. Finally, Section V concludes the paper.

\section{RELATED WORK}

Existing analytical studies of P2P streaming model have shown some potential improvements in its performance. Previous literatures about P2P streaming systems mostly focus on improving the performance within a single channel $[1,2]$. However Hei et al. [3, 4] investigated the distribution of peer life time in PPLive, and found P2P streaming systems generally provide lower streaming QoS in small group channel compared to large popular channel. Zimu Liu [5] characterized the peer lifetime patterns in unpopular channels. It further indicates that promoting peer stability will be better for streaming quality.

Recently a few multi-channel related works have been published. $\mathrm{Wu}$ et al. [6] proposed the View-Upload Decoupling (VUD) approach to bring stability to multichannel and enabling cross-channel resource sharing. Gan et al. [7] proposed a reputation-based incentive mechanism to stimulate peers in performance-rich channels to help peers in performance-poor channels.

However, some fundamental facts that have important influence on the channel design are not well described. In this paper, we propose a cross-channel federation model to address these challenges. By investigating the identification of stable nodes and buffering-progress-based peering, we address the Cross-channel federation Construction and Optimization. With proper peer assignment, our system has demonstrated the efficiency and robustness in simulation. 


\section{CROSS-CHANNEL FEDERATION MODEL}

We consider a live video streaming system with multichannels. The video stream, originated from the source, are divided into equal-length blocks and distributed into different channels. The peers that are interested in the same channel form a mesh-based channel group and can join and leave the channel at will. Our cross-channel federation model allows peers to join multi-channel. Our goal is to identify a set of nodes having superabundant abilities in popular channel to assist the unpopular channel.

\section{A. Model Cross-channel federation}

Due to such isolated-channel model, resources are scarce in unpopular channels with unbalanced peer population. Peers frequently churn and their residence time is too short, which further aggravate the decrease of QoS in the channels. Isolated-channel model has become one of the important reasons for blocking current $\mathrm{P} 2 \mathrm{P}$ streaming media system with its native limitation. Cross-channel collaboration model has provided an opportunity to improve P2P system performance. In cross-channel model peers are not isolated in one channel. They are allowed to joint multi-channel. It seems we can choose some peers in popular channel to join the broadcast in unpopular channel and help to improve the performance.

Based on above consideration, we setup the superabundant federation model which can be viewed as a specific cross-channel mode. In such a federation model, only nodes having superabundant abilities can be selected as the helper. This mode can not only increase the performance of the unpopular channel but also minimize the impact of original channel.

To model our domain, we assume that there are a set of users $\mathrm{U}=\left\{u_{1}, u_{2}, \ldots, u_{n}\right\}$ and a set of channels $\mathrm{CH}=\left\{c h_{1}, c h_{2}, \cdots, c h_{n}\right\}$. We define $\mathrm{P}(\mathrm{CH})$ as the power set of $\mathrm{CH}$, that is, the set of all subset of $\mathrm{CH}$. If $\mathrm{CH}$ is a finite set with $|C H|=k$ elements, then the power set of $\mathrm{CH}$ contains $|P(C H)|=2^{n}$ elements. Define function: $f_{c h}: \mathrm{U} \rightarrow \mathrm{P}(\mathrm{CH})$, which maps users to channels. Then $f_{c h}\left(u_{i}\right)$ is the set of channels which user $u_{i}$ shares. It is clear that $U_{i}=\sum_{i=1}^{n} f_{c h}\left(u_{i}\right)=\mathrm{CH} .\left|f_{c h}\left(u_{i}\right)\right|$ must be greater than zero. If $\left|f_{c h}\left(u_{i}\right)\right|=0, \quad u_{i}$ doesn't joint any channel. If $f_{c h}\left(u_{i}\right)=f_{c h}\left(u_{j}\right)$, user $i$ and user $\mathrm{j}$ have the same interesting channels. $f_{c h}\left(u_{i}\right) \cap f_{c h}\left(u_{j}\right)=\phi$ indicates that user $i$ and user $\mathrm{j}$ belong to different channel. Watching a certain channel $\mathrm{j}$ at time $t$ form the users' point of view, we have set $U_{j}(t)=\left\{u_{i}(t) \mid f_{c h}\left(u_{i}(t)\right)=c h_{j}(t), \mathrm{i}=1, \ldots \mathrm{n}\right\}$.

Architecture comparison between Isolated-Channel and Federation-Channel is shown in Figure 1.

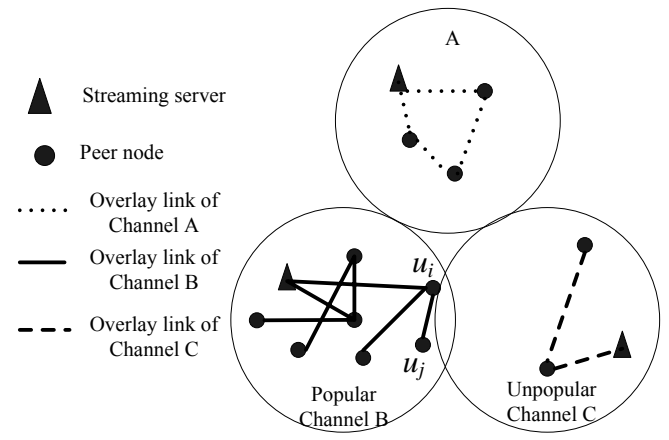

(a)

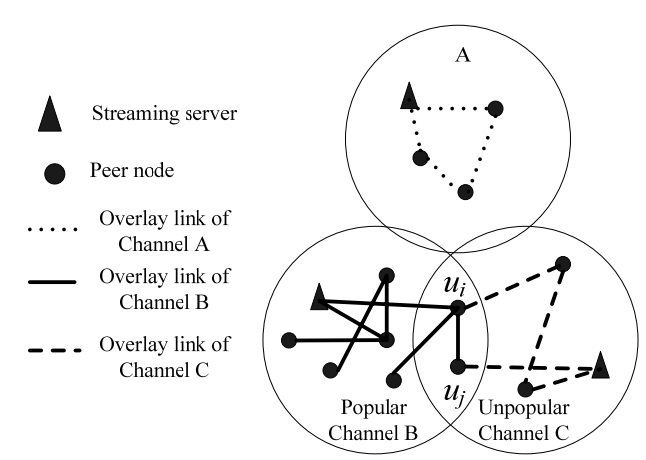

(b)

Figure 1. Architecture comparison between Isolated-Channel and Federation-Channel: (a) A three isolated-channel model (b) A three crosschannel federation model

Suppose there are three Channel P2P systems. Channel B is a popular channel with nodes more than 100. Channel A and Channel $\mathrm{C}$ is unpopular channel only having 4 nodes and 3 nodes respectively. From Figure 1(a) we have $\left|f_{c h}\left(u_{k}\right)\{\mathrm{k}=1, \ldots, \mathrm{n}\}\right| \quad=1, \quad U_{A}(t) \cap U_{B}(t)=\phi$, $U_{A}(t) \cap U_{C}(t)=\phi, U_{B}(t) \cap U_{C}(t)=\phi$. Obviously, these three channels are separately managed in isolated-channel mode. Channel A and C Only have a few nodes and the resources are scarcely.

Figure $1(\mathrm{~b})$ is the federation model where peers can joint multi-channel performance. $\mid f_{c h}\left(u_{k}\right) \quad\{\mathrm{k}=1, \ldots, \mathrm{n}$ and $\mathrm{k} \neq \mathrm{i}, \mathrm{j}\}|=1, \quad| f_{c h}\left(u_{k}\right) \quad\{\mathrm{k}=\mathrm{i}, \mathrm{j}\} \mid=2, \quad U_{A}(t) \cap U_{B}(t)=\phi$, $U_{A}(t) \cap U_{C}(t)=\phi, U_{B}(t) \cap U_{C}(t)=\left\{u_{i}, u_{j}\right\} . u_{i}$ and $u_{j}$ in popular channel $\mathrm{B}$ are joined in the broadcast in unpopular channel C. They can improve the performance of channel C.

\section{B. Streaming Quality}

Assume that nodes' pure altruism periods have the negative exponential distribution with mean value $1 / \alpha$. Similarly, nodes' pure egoism periods can be described by a random variable exponential distribution with mean value $1 / \beta$ and nodes' altruism-egoism periods with mean value $1 / \gamma$. Therefore, the probability that a node is on altruism can be given by $P_{\text {altruism }}=(\alpha+\gamma) /(\alpha+\beta+\gamma)$. Since the nodes have 
independent distribution, the probability that node $i$ is altruism can be given by

$$
P(i, N)=\left(\begin{array}{c}
i \\
N
\end{array}\right) P_{\text {altruism }}^{i}\left(1-P_{\text {altruism }}^{i}\right)^{N-i}
$$

Then, the expected value of node $i$ which following $P(i, N)$ distribution can be calculated by

$$
\mathrm{E}[P(i, N)]=\mathrm{E}\left[\left(\begin{array}{c}
i \\
N
\end{array}\right) P_{\text {altruism }}^{i}\left(1-P_{\text {altruism }}^{i}\right)^{N-i}\right]=\mathrm{N} P_{\text {altruism }}
$$

Equation (2) calculates the average number of altruism nodes.

To evaluate the channel performance, we express the resource performance of channel $\mathrm{j}$ as

$$
P_{j}=\frac{S_{j}+U_{j}\left(H_{j}\right)}{D_{j}\left(M_{j}\right)}=\frac{S_{j}+\sum_{i=1}^{H} u_{j}^{i}}{\sum_{i=1}^{M} d_{j}^{i}}
$$

Where $S_{j}$ is the total supported bandwidth available for the channel from server, $U_{j}\left(H_{j}\right)$ is the total upload bandwidth provided by the peers which given the number of $\mathrm{H}$ altruism nodes in channel j. $D_{j}\left(M_{j}\right)$ is the total download required bandwidth which given the number of $M$ egoism nodes. As the resource performance of channel $\mathrm{j}, P_{j}$ describes the degree whether the upload supply can match the download demand for the channel. If $P_{j} \geq 1$, the upload steaming can sustain the download requirement. Higher $P_{j}$ have better streaming quality with lower delays, better playback and scheduling insensitive.

According to (3) we have

$$
P_{c}=\frac{S_{c}+N_{c} P_{\text {altruism }} \bar{u}_{c}+\operatorname{Service}\left(u_{i}+u_{j}\right)}{N_{c} P_{\text {egoism }} \bar{d}_{c}}
$$

As we have known, $P_{c}$ describes the degree whether the upload supply can match the download demand for channel C. Compare to Figure1(a), channel $\mathrm{C}$ in federation model has increase the upload supply $\operatorname{Service}\left(u_{i}+u_{j}\right)$ in Figure1(b).

Therefore we get higher $P_{c}$, which means we have better streaming quality.

\section{PERformance Evaluation}

\section{A. Methodology}

To evaluate the performance of cross-channel federation model, we have implemented an extensive experiment based on NS2 simulator.
We construct a network topology with 1024 peers in the experiment. Peers are organized into popular channel with more than 100 nodes and unpopular channel with less than 30 nodes. Within each group, the channel peers are organized into a mesh network and use Push-Pull method for chunk scheduling. There exist channel streaming servers, which help to maintain the neighborhood of a channel and continuously transfer the streaming chunk into randomly selected channel peers. To join a channel, the new peer should contact the channel streaming server and get the channel neighbor list. Then all other tasks, including downstream peer selection and data exchanges, are performed locally at peers.

\section{B. Simulation Results}

In the simulation, we adopt a dynamic scenario for the evaluation, where peers arrive and departure at the system following the Poisson process. In the default setting, after staying at the channel 200 time slots where the system enters steady state, peers start to switch their channels. The session length $\mathrm{L}$ is set to 1000 time slots and each data block is of 1second video. In the following part, we present the experimental results obtained from our simulation. In convenience $\mathrm{T}$ refers to threshold time $\mathrm{T}(\mathrm{t}), \mathrm{B}$ refers to buffer level.

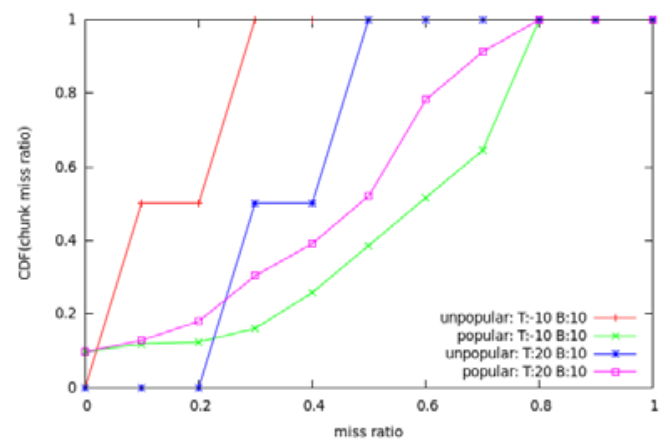

Figure 2. CDF of Chunk Miss Ratio with threshold time -10 and 20

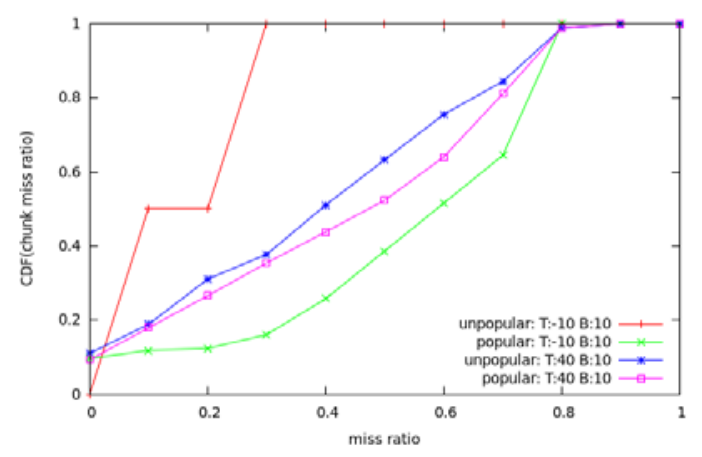

Figure 3. CDF of Chunk Miss Ratio with threshold time -10 and 40

In Figure 2 and Figure 3, we plot the CDF of churn miss rates under different threshold and the same buffer level. From these figures we observe that threshold time has important influence on the performance of unpopular channel. When threshold time is set to -10 slot, it causes more peers in popular channel to be identified as stable 
nodes and be selected for assisting. Although the initial CDF of unpopular channel is less than popular channel, it grows quickly under the help of popular channel. There is not much difference for peers in different channels in terms of chunk miss ratio.

\section{CONCLUSIONS}

This paper presents a cross-channel federation model to accelerate unpopular channel. The key idea is to identify a set of stable nodes in popular channel to assist the unpopular channel. These stable nodes, together with nodes in unpopular channel, are organized as mesh-based network, which facilitates the unpopular channel to enlarge the channel group and promote peer stability. Simulations based on NS2 have been setup to evaluate our federation model. In the future work, we will focus on adding adaptive Peer Assignment for constructing our cross-channel federation model.

\section{ACKNOWLEDGMENT}

This work is supported by "CDIO-based data engineering research and implementation" (Grant No. Rj010), and
National Natural Science Foundation of China (Grant No. 61263043).

\section{REFERENCES}

[1] Tang, Y., Sun, L.F., Zhang, K.Y., Yang, S.Q., Zhong, Y.Z.: Longer, better: On extending user online duration to improve quality of streaming service in P2P net-works. In: Proc. IEEE ICME '07. (July 2007) 2158-2161.

[2] Stutzbach, D., Rejaie, R.: Understanding churn in peer-to-peer networks. In: Proc. ACM IMC '06. (2006) 189-202

[3] Hei, X., Liu, Y., Ross, K.W.: Inferring network-wide quality in P2P live streaming systems. IEEE Journal on Selected Areas in Communications (JSAC) 25(9) (2007) 1640 - 1654.

[4] Hei, X., Liang, C., Liang, J., Liu, Y., Ross, K.: A measurement study of a large-scale P2P IPTV system. EEE Transactions on Multimedia 9(8) (2007) 1672-1687.

[5] Zimu Liu, Chuan Wu, Baochun Li , Shuqiao Zhao. Why are Peers Less Stable in Unpopular P2P Streaming Channels? In: Networking. (2009) 274-286.

[6] D. Wu, C. Liang, Y. Liu, and K. W. Ross, View-upload decoupling: A redesign of multi-channel $\mathrm{P} 2 \mathrm{P}$ video systems. in Proceedings of IEEE INFOCOM, Mini-Conference, Rio de Janeiro, Brazil, 2009, pp. $1-6$.

[7] G. Tan and S. Jarvis, Inter-Overlay Cooperation in High-Bandwidth Overlay Multicast. in ICPP-06, Aug. 2006. 